\title{
Gas-Induced Drying of Nanopores
}

\author{
Gaia Camisasca, ${ }^{\ddagger}$ Antonio Tinti, ${ }^{\ddagger}$ and Alberto Giacomello*
}

Cite This: J. Phys. Chem. Lett. 2020, 11, 9171-9177

Read Online

ABSTRACT: Here, we investigate the role of a dilute hydrophobic gas on the phase behavior of water confined in hydrophobic nanopores. Molecular dynamics showed that gas atoms are hydrophobically attracted within the pores, where even a single particle is able to induce spontaneous drying of the whole pore. The drying process is rationalized in terms of its free-energy landscape, revealing that the penetration of a gas atom is able to suppress the drying free-energy barriers of hydrophobic pores. Results provide insights into the role of gases on the wettability of nanopores and evidence of a possibile physical mechanism for the action of volatile anesthetics on some kinds of ion channels. Results also indicate a novel, bioinspired strategy for controlling bubble formation in nanopores for sensing and energy

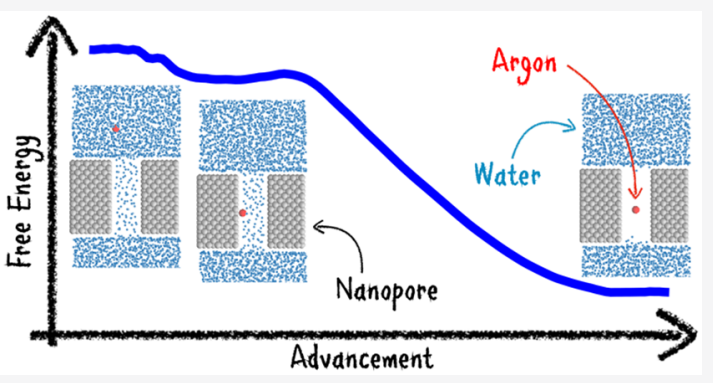
applications.

$\mathrm{T}$ he phase behavior of water in nanoscale pores is of utmost importance for a number of phenomena in biology and technology. Among the former, evidence is growing that the functioning of ion channels (the gates of cellular membranes) often relies on the evaporation of water inside the pore which is able to block (gate) ionic currents through it. ${ }^{1-6}$ In the realm of technology, nanoporous materials are showing promise for a number of applications, 7,8 including energy technologies ranging from mechanical energy storage to its dissipation. ${ }^{9}$ Understanding how the physical and chemical characteristics of nanopores determine their wetting/ drying conditions enables their rational design. ${ }^{10}$ At the boundary between biology and technology, biomimetic nanopores $^{11-13}$ are emerging for sensing biomolecules ${ }^{14,15}$ and to sequence DNA. ${ }^{16}$ In brief, understanding and controlling the phase behavior of water, or more generally, of liquids, in extreme confinement has critical consequences on a number of issues in biomedicine, (bio)nanotechnology, and fundamental physics. ${ }^{17}$

Equilibrium microscopic density functional theory calculations have been used in the past to show that small concentrations of homogeneously dissolved hydrophobic gases $^{3}$ energetically favor states in which vapor and gas occupy the inner section of a hydrophobic nanopore (see also ref 18), effectively rendering the channel non conductive to ionic currents. In this regard, the effect of a hydrophobic dissolved gas is not dissimilar from the effect of negative liquid pressures or increased hydrophobicity and/or confinement. The present scenario is important for a number of reasons, one being that in many experiments dissolved gases are difficult to eliminate and can thus cause unwanted drying, e.g., in solid-state nanopores for sensing. ${ }^{2,19}$ This intriguing phenomenology is also supposed to be related to the clinical usage of inert gases, and in particular of noble gases such as xenon, as general anesthetics. The modern understanding of general anesthesia suggests that this complex phenomenon is mediated by a number of molecular mechanisms, ${ }^{20}$ including the direct or indirect action of gases on certain classes of ion channels. ${ }^{21}$ Interestingly, there is structural evidence of xenon binding to the pore domain of pentameric ligand-gated channels, ${ }^{22}$ which is compatible with the mechanism discussed below.

The goal of this work is to observe and rationalize the microscopic mechanism of drying in the presence of a dilute hydrophobic gas in order to achieve the much desired control of bubble formation in nanoscale confines. The physical mechanism identified here is general enough to provide a possible explanation for the action of volatile anesthetics and suggest bioinspired strategies for technological applications.

The current work leverages advanced molecular dynamics (MD) simulations to reveal the mechanism and assess the kinetics of drying in a nanopore in the presence of a single hydrophobic atom. We consider a thought experiment in which a gas is added in small quantities to the liquid phase far from the nanopore. In this dilute regime, the effect of a single particle on the wetting state of the pore is assessed by computing the free-energy landscape for drying and the most probable paths joining the fully wet to the dry states. It is found that, in the absence of the particle, drying is a thermally activated event characterized by a free-energy barrier larger

Received: August 25, 2020

Accepted: October 8, 2020

Published: October 15, 2020 


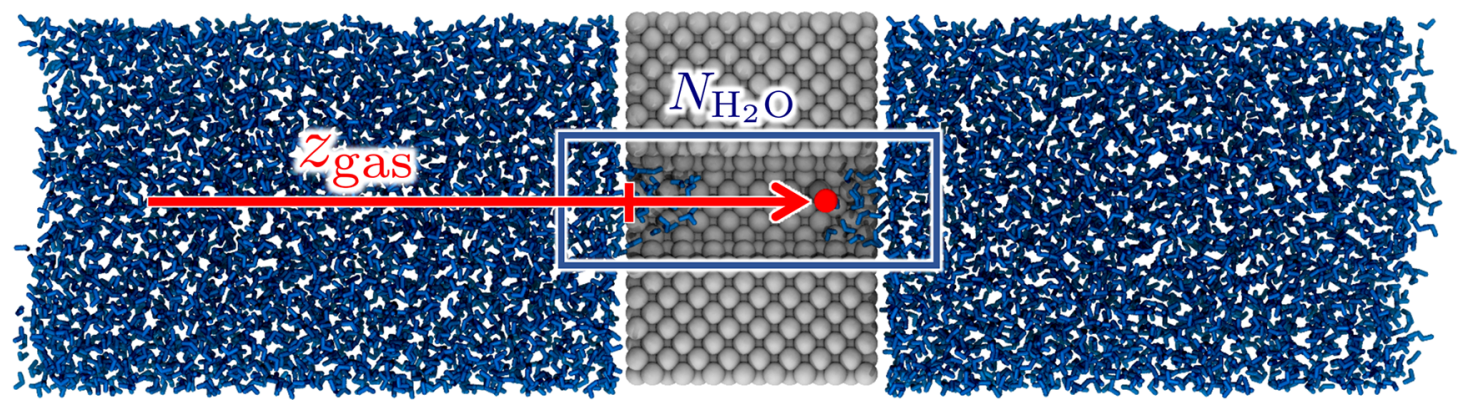

Figure 1. System used in MD simulations, comprising a solid wall from which a cylindrical pore is excavated. The solid is immersed in SPC/E liquid water; an argon atom is added in the fluid domain (in red, larger than actual scale). The two descriptors used in RMD (axial gas coordinate and number of water molecules inside a parallelepipedic domain enclosing the pore) are also defined.
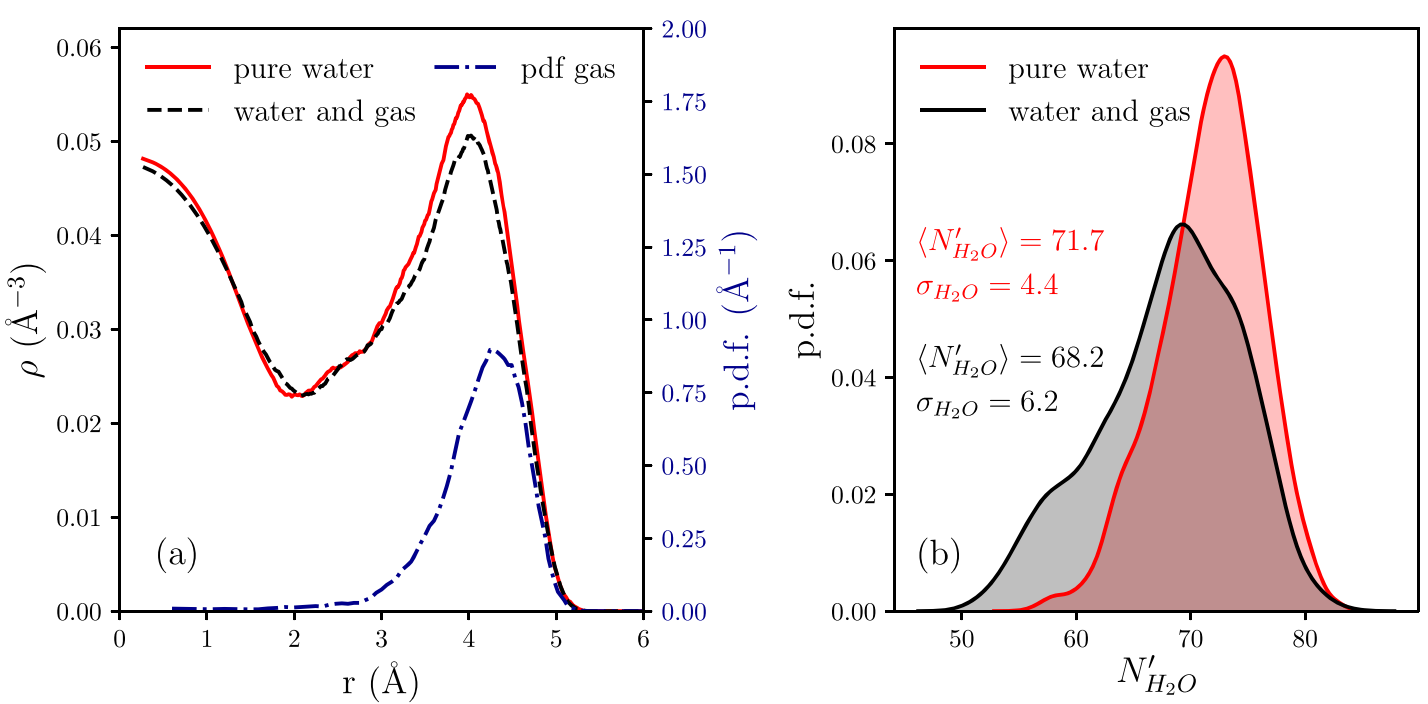

Figure 2. (a) Density of water within the pore as a function of the radius from unrestrained MD simulations in the presence (black dashed line) or in the absence of the gas atom (red solid line). The probability density function for the radial position of the gas atom is shown with a blue dashdotted line. Wall is located at $r \approx 7 \AA$. (b) Histogram of the number of water molecules inside the nanopore, $N_{\mathrm{H}_{2} \mathrm{O}}^{\prime}$, with (black) and without (red) the gas atom computed from unrestrained MD simulations in the wet state. The black curve is obtained from configurations in which the gas atom is outside the pore; the red curve from configurations with the gas inside the pore (and before drying).

than the thermal energy $k_{\mathrm{B}} T$. Surprisingly, we found that even a single hydrophobic atom is able to abate the drying freeenergy barrier, causing the instantaneous drying of the nanopore.

We consider a cylindrical pore with diameter $14 \AA$ excavated from an $f c c$ Lennard-Jones crystal, Figure 1 . The pore has a length of $26 \AA$ and is immersed in SPC/E water. ${ }^{23}$ While the dimensions of the pore have been chosen in order to approximately reproduce the typical size of the sections of ion channels that exhibit gating, ${ }^{3}$ the interaction potential and the structure are kept purposely generic as we aim at obtaining an understanding of the overarching physical mechanics of drying in the presence of gas particles. Water interactions with the pore are of the Lennard-Jones kind, and the hydrophobicity of the surface is tuned by multiplying the attractive term by a constant $c=0.98$, rendering a Young contact angle $\theta_{Y}=104^{\circ} \pm 2^{\circ}$; see the Supporting Information for details on contact angle calculations. This value is chosen to be comparable with the typical hydrophobicity of nonpolar amminoacids. ${ }^{24}$ A less hydrophobic pore with $\theta_{Y}=93^{\circ}$ is also considered in order to demonstrate that the identified mechanism is rather general and could apply to a variety of biological and synthetic environments. A single argon particle is added to water, ${ }^{a}$ as defined by its Lennard-Jones interactions with water and with the pore walls. ${ }^{25}$ As further discussed in the Supporting Information, the chosen interaction makes this particle poorly soluble ("hydrophobic"). We will sometimes refer to this atom as "gas" to remember that we aim at reproducing a specific experiment in which a poorly soluble gas is added in the liquid phase; however, the following results also apply to other scenarios in which a hydrophobic particle enters a nanopore.

The pressure in the liquid phase is controlled by leaving a free liquid-vapor interface at both ends of the simulation box far from the nanopore; this procedure is used to accurately produce liquid-vapor coexistence. $^{26}$ The temperature is controlled by a Nosé-Hoover chains thermostat. ${ }^{27}$ We refer to the Supporting Information for a complete overview of the setup and methods used in the simulations.

In order to illustrate the effect of the gas particle on the wetting state of the nanopore, long, unrestrained $\mathrm{MD}$ simulations are conducted, starting from configurations with the gas atom in the liquid bulk far from the nanopore. In most cases, the gas atom explores the bulk liquid region, yet a number of realizations eventually exhibit the relevant phenomenology; i.e., the gas particle approaches the solid 

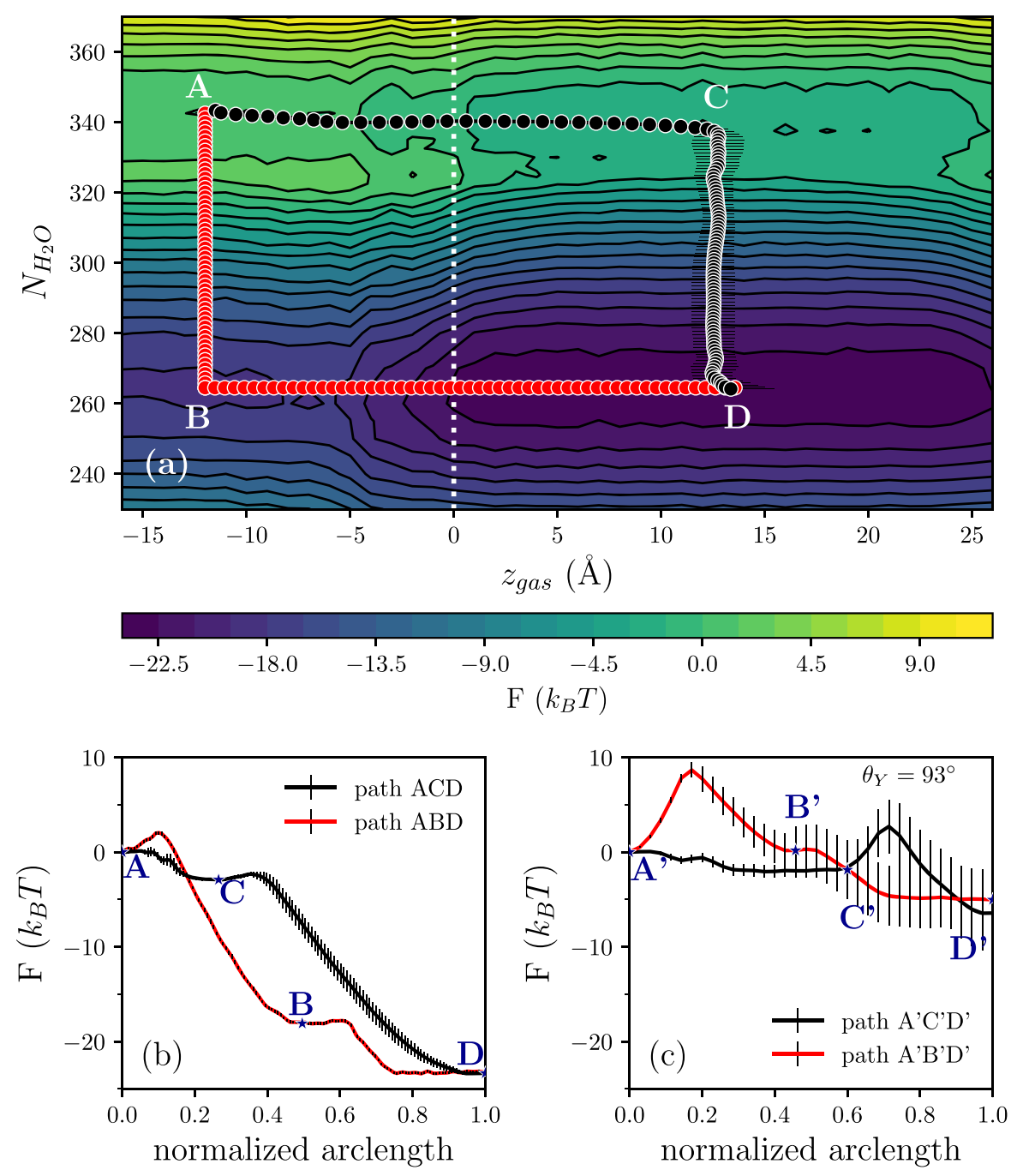

Figure 3. (a) Free-energy map for the drying of the nanopore with $\theta_{Y}=104^{\circ}$ as a function of the number of water molecules inside the control region defined in Figure 1 and of the axial position of the argon atom. The nanopore is located at $0<z_{\text {gas }}<26 \AA$; the pore entrance corresponds to the white dotted line. The two paths discussed in the main text are shown; the related free-energy profiles are reported in (b) as a function of the normalized arclength (same color code). (c) Free energy along analogous paths for the pore with $\theta_{Y}=93^{\circ}$. Primed letters indicate the four main states of this pore. Error bars were estimated according to the procedures discussed in the Supporting Information. An arbitrary constant is added to the free-energy so that it vanishes at states $A$ and $A^{\prime}$.

domain and then enters the pore where it induces drying, as shown in the Supporting Information video.

Poorly soluble gases are known ${ }^{28}$ to be attracted to flat hydrophobic walls, where they minimally disrupt the water structure. The more hydrophobic the interactions between the liquid and the wall, the more the gas is attracted to the latter. Gas solubility has a similar effect, with poorly soluble gases preferentially concentrating at the walls. In addition to these generic effects, the cylindrical geometry is here found to funnel the gas inside the pore where it remains trapped for extended periods of time. There, the atom preferentially resides close to the wall, decreasing the local water density ${ }^{b}$ (Figure 2a).

MD simulations reveal an important qualitative effect produced by the hydrophobic particle, associated with the enhancement of water density fluctuations within the pore. As the gas particle enters the pore, several intense fluctuations, which deplete the local liquid density, are observed within the pore (Figure $2 b$ ). It is seen that the presence of the gas atom decreases the average water occupancy by four molecules, more than expected from the size of argon alone. More prominently, the tails of the distribution are amplified by the presence of the gas atom, specifically those corresponding to large density depletions. Indeed, these fluctuations culminate in the drying of the pore, when a sufficiently large fluctuation occurs; i.e., a "bubble" forms with size comparable to the pore diameter (see the Supporting Information video). In short, these simulations show that the gas atom has a double action: it is hydrophobically attracted within the pore and there it "catalyzes" drying by enhancing density fluctuations of water.

In order to rationalize the previous observations we quantified the catalytic action of a gas particle and determined the drying mechanism(s) by MD free-energy calculations. The free-energy landscape for drying of the system in Figure 1 was computed as a function of two descriptors: the position of the gas atom projected on the pore axis, $z_{\text {gas }}$ and the number of water molecules inside a control region enclosing the pore, $N_{\mathrm{H}_{2} \mathrm{O}}$ (see Figure 1). These variables control the penetration of gas particles into the pore and the wetting/drying process of the pore. A rare-event method, Restrained Molecular Dynamics, ${ }^{29}$ is used, which allows us to efficiently sample 
even improbable regions of the phase space connected to freeenergy barriers; in brief, the method consists in adding harmonic restraining potentials to the physical one, which enable the computation of the partial derivatives of the free energy; see the Supporting Information for details. The freeenergy landscape is then computed by integrating the freeenergy gradient in the two variables using a specialized numerical technique, known as Global Least Squares method. $^{30}$

Figure 3a shows a color map of the two-dimensional freeenergy landscape. It is seen that two main horizontal "valleys" are present: the uppermost corresponding to a pore filled with water and the lowermost to an empty pore. This feature is in agreement with previous studies of wetting/drying of nanopores. ${ }^{31}$ However, because of the presence of the gas particle, these valleys descend from left to right, forming two plateaus, corresponding to the argon atom outside and inside of the pore, respectively. Except for some smooth decrease of the free-energy in correspondence of the argon entering through the cavity mouth, its translation inside the pore has no effect on the free energy, which remains flat. Physically, this freeenergy landscape corresponds to a multistable system, with four main states: filled pore/argon in the bulk (A), empty pore/argon in the bulk (B), filled pore/argon in the pore (C), and empty pore/argon in the pore (D). Due to the pore hydrophobicity and to the extreme confinement, the filled states are metastable; i.e., they have higher free energy than the empty ones. When inside the pore, argon decreases the free energy of both the empty and filled state by 5 and $3 k_{\mathrm{B}} T$, respectively: this is a quantification of its hydrophobic attraction to the pore. The combined action of pore hydrophobicity and of the presence of a hydrophobic gas makes point $\mathrm{D}$ the global free-energy minimum, i.e., the thermodynamically stable state.

The free-energy landscape of Figure 3a, allows us to discuss the drying mechanisms. We consider an ideal experiment that is started from the filled pore and in which a small concentration of gas is added far from the pore; the final state of this experiment is the thermodynamically stable state, i.e., the empty pore with argon in it. This experiment corresponds to finding a line joining points A and D in Figure 3a. The relevant path is the most probable one: the minimum free-energy path. We use the simplified string $\operatorname{method}^{32}$ in order to identify it. Actually, depending on the initial conditions, more than one path that locally minimizes the path free energy is possible; see for instance the drying paths in a pore. ${ }^{33}$ We discuss here the two main paths relevant for this system, i.e., one in which the pore empties and, subsequently, the gas enters the pore $(\mathrm{ABD}$, red) and one in which argon enters the pore and then the pore empties (ACD, black); see also Figure $3 b$ reporting the free energies along these two paths. The first path is characterized by a free-energy barrier of ca. $2 k_{\mathrm{B}} T$ necessary to accomplish the initial emptying of the pore; this barrier is connected to the free-energy cost of forming a critical bubble. ${ }^{31}$ There is instead no barrier associated with the entry of argon in the already empty pore. Importantly, the second path has vanishingly small barriers $\left(0.6 k_{\mathrm{B}} T\right)$, with two extended plateaus corresponding to the argon entering the pore and to its subsequent emptying: a single hydrophobic atom is able to effectively suppress the drying free-energy barrier, destabilizing the filled state.

In order to check the generality of gas-induced drying, we also simulated a solid that is approximately neutral to wetting,
$\theta_{Y}=93^{\circ} \pm 2^{\circ}$ (Figure $3 \mathrm{c}$ ), where the relative thermodynamic stability of the initial and final states of our experiment is preserved. Similar results apply also to hydrophilic pores, for which, however, the filled state is the stable one. We directly computed the free energy along two paths analogous to those in Figure $3 a$ identified as $\mathrm{A}^{\prime} \mathrm{C}^{\prime} \mathrm{D}^{\prime}$ and $\mathrm{A}^{\prime} \mathrm{B}^{\prime} \mathrm{D}^{\prime}$. Interestingly, the phenomenology remains qualitatively the same: the entry of the gas in the wet pore accelerates drying as compared to the case in which pore dewets first. The main quantitative difference is that the drying barriers are generally higher, as expected because of the less hydrophobic walls; gas decreases the free-energy barriers from 8.7 to $4.6 k_{\mathrm{B}} T$, even more prominently than in the case with $\theta_{Y}=104^{\circ}$. As in the previous case, there is no barrier for the entrance of the gas in the pore both in the filled and in the empty states; the free-energy gain for these events is 2 and $5 k_{\mathrm{B}} T$, respectively; i.e., there is a significant attraction of the gas inside the pore.

We are now in a position to discuss the generic physical effect of diluted hydrophobic gases or hydrophobic particles on the stability of confined liquid water. Due to their hydrophobicity, gas particles tend to migrate to interfaces, in particular, inside the pore. There, gas particles enhance water density fluctuations, acting as nucleation seeds at the walls where they preferentially reside. This is precisely the location at which critical nucleation bubbles form, due to the favorable hydrophobic environment. ${ }^{31,34,35}$ Such gas-induced fluctuations are thus synergic with the facilitated drying occurring in hydrophobic nanopores, being capable of effectively decreasing (and, for the highest hydrophobicity, suppressing) the freeenergy barrier.

Interestingly, the reported drying mechanism is in principle independent of the gas concentration in the bulk, as it requires only one hydrophobic atom. However, the initial plateaus in the free-energy profiles in Figure 3 conceal the step of diffusion of the gas to the pore, which depends on its bulk concentration and is instrumental for gas-induced drying itself. One can thus break down the overall kinetics of the process of gas-induced drying in two main steps: diffusion to the pore and pore drying itself. Under the reasonable hypothesis that the two processes are independent, the average drying time is given by the sum of the average times of the two processes. For the first, a steadystate estimate of diffusion to a circular target exists, ${ }^{36}$ whereas for the latter one expects an Eyring-like equation, yielding

$$
t_{\text {tot }}=t_{\text {diff }}+t_{\text {dry }} \approx \frac{1}{4 \pi D C_{\infty} r}+t_{0} \exp \left(\Delta F^{\dagger} /\left(k_{\mathrm{B}} T\right)\right)
$$

where $D$ is the diffusion coefficient of argon in water, $C_{\infty}$ is the bulk gas concentration, $r$ is the pore radius, $t_{0}$ is a kinetic prefactor, and $\Delta F^{\dagger}$ is the drying free-energy barrier in the presence of gas. Using $D=3 \times 10^{-5} \mathrm{~cm}^{2} / \mathrm{s}, C_{\infty}=15 \mathrm{mM}$, and $r=7 \AA$, we get $t_{\text {diff }} \approx 8$ ns. We estimate $t_{0} \approx 2$ ns from the unrestrained simulation. In the case of vanishing barrier $\left(\theta_{Y}=\right.$ $\left.104^{\circ}\right), t_{\mathrm{dry}} \approx t_{0}$ and thus $t_{\text {tot }} \approx 10 \mathrm{~ns}$, with diffusion being slightly slower than drying itself. For $\theta_{Y}=93^{\circ}$, instead, drying becomes the kinetic bottleneck with $t_{\text {dry }} \approx 25 t_{\text {diff. The }}$ concurrent presence of multiple gas particles in the nanopore relevant at higher gas concentrations falls outside the scope of this Letter and will be addressed in an upcoming work. Overall, the gas changes the kinetics of drying, abating the free-energy barriers, and also its thermodynamics, further stabilizing the empty state.

In the context of general anesthesia, there is a century-old empirical correlation between the anesthetic potency and the 
hydrophobicity of anesthetic gases: the so-called MeyerOverton correlation, whose traditional interpretation is that the lipid bilayer is the likely location of anesthetic action. ${ }^{37}$ The correlation applies at clinically relevant concentrations even for noble gases, such as xenon, which typically have a low affinity for biological targets. ${ }^{38}$ More recently, it has been recognized that ion channels are the more likely target of general anesthetics; ${ }^{37}$ in particular, it has been proposed that "inert" anesthetics may block some ion channels via the hydrophobic gating mechanism, i.e., by drying of the pore domain aided by hydrophobic gases. ${ }^{3}$ Our simulations indeed support the existence of such a gas-assisted gating mechanism in the hydrophobic (or even neutral) portion of channels, the hydrophobic gate, ${ }^{5}$ which occurs in a variety of ligand and voltage gated channels of potential relevance for anesthesia. ${ }^{39}$ Given the generality of our model, the mechanism is completely nonspecific, which would explain the variety of hydrophobic gases that serve as general anesthetics. In addition, the present results provide a tempting interpretation on why the enhanced anesthetic potency correlates with the hydrophobicity of the gas, i.e., because it increases the attraction of the gas inside nanopores and accelerates the hydrophobic gating process of ion channels.

In the light of the present results, one expects that the presence of hydrophobic gases in a liquid can significantly affect the wetting state of biomimetic artificial nanopores, which is crucial, e.g., in nanotechnology applications. An important case is nanopore sensing ${ }^{14}$ and sequencing, the latter of which is already successfully employed for DNA. ${ }^{40}$ In such applications, the formation of bubbles inside nanopores should be avoided in order to lower the noise ${ }^{2,19}$ in the measurement of ionic currents, from which the type of analyte, e.g., the nucleotide, is determined. Current results suggest that, in order to achieve a better sensitivity and reproducibility in nanopore technologies, it is crucial to control the amount of gas dissolved in the liquid during the experiment. In addition, although hydrophobic gases always accelerate the formation of bubbles, more hydrophilic pores are less prone to this phenomenon and are thus preferable as templates for sensing. Again, since the present results apply to a variety of nanopores with different levels of hydrophobicity, the caveat applies both to solid-state nanopores ${ }^{41}$ and to bioengineered pores. ${ }^{42}$

In other technological contexts, hydrophobic gases may provide an opportunity to control the wetting state of nanostructured surfaces, facilitating, for instance, the recovery of superhydrophobic states. ${ }^{43}$ The findings hereby reported may also have a significant impact in the domain of artificial nanopore technology; e.g., they may be useful in energy applications of nanoporous materials. ${ }^{44,45}$ In this latter field, it is crucial to control the intrusion and extrusion pressures and their hysteresis, determining the performance and applicability of the devices. Adding a hydrophobic gas provides an efficient way to facilitate liquid extrusion from nanopores, increasing the extrusion pressure; on the other hand, the intrusion process involves the advancement of the liquid front into the pore, which is weakly affected by the presence of a gas particle in the empty pore; therefore, the intrusion process is expected to occur at similar pressures in the presence or in the absence of gas. The presence of gas particles thus acts asymmetrically on the intrusion and extrusion processes, ${ }^{3}$ with a greater sensitivity for the extrusion process, leading to a decrease of the intrusion/extrusion hysteresis. A more complete discussion on the role of hydrostatic pressure and of the particle size is deferred to future work.

In conclusion, molecular dynamics simulations have allowed us to observe the peculiar physics of drying of nanopores induced by hydrophobic particles dissolved in the liquid: gas atoms are hydrophobically attracted inside the nanopore where they increase water density fluctuations, promoting drying. Free-energy calculations quantified this catalytic action and exposed the generic mechanism by which the system circumvents the usual drying path. For a variety of wall wettabilities, ranging at least from hydrophobic to neutral, the presence of a poorly soluble gas particle was shown to effectively catalyze drying. Simulations further showed that even extremely low gas concentrations can achieve this effect (in principle, even a single hydrophobic particle) in very rapid time scales. These results reveal a new route to control the wetting and drying processes in extreme confinement, with important applications in the field of general anesthetics, nanopore sensing, nanoporous material for energy applications, and superhydrophobic surfaces.

\section{ASSOCIATED CONTENT}

\section{st Supporting Information}

The Supporting Information is available free of charge at https://pubs.acs.org/doi/10.1021/acs.jpclett.0c02600.

Methods, simulation details, free-energy calculations via RMD and error estimation, contact angle estimates, characterization of gas hydrophobicity (PDF)

Video of MD trajectory showing spontaneous drying of the pore induced by the entrance of the gas particle inside the pore (MP4)

\section{AUTHOR INFORMATION}

\section{Corresponding Author}

Alberto Giacomello - Dipartimento di Ingegneria Meccanica e Aerospaziale, Sapienza Università di Roma, 00184 Rome, Italy; (i) orcid.org/0000-0003-2735-6982; Email: alberto.giacomello@uniroma1.it

\section{Authors}

Gaia Camisasca - Dipartimento di Ingegneria Meccanica e Aerospaziale, Sapienza Università di Roma, 00184 Rome, Italy; (1) orcid.org/0000-0002-0789-2641

Antonio Tinti - Dipartimento di Ingegneria Meccanica e Aerospaziale, Sapienza Università di Roma, 00184 Rome, Italy

Complete contact information is available at:

https://pubs.acs.org/10.1021/acs.jpclett.0c02600

\section{Author Contributions}

${ }^{\ddagger}$ G.C. and A.T. contributed equally to this work

\section{Notes}

The authors declare no competing financial interest.

\section{ACKNOWLEDGMENTS}

This research is part of a project that has received funding from the European Research Council (ERC) under the European Union's Horizon 2020 research and innovation programme (grant agreement No. 803213). The authors acknowledge PRACE for awarding us access to Marconi100 at CINECA, Italy and to Joliot-Curie at GENCI@CEA, France. 


\section{ADDITIONAL NOTES}

${ }^{a}$ Considering one argon atom dissolved in the volume of one liquid reservoir, the resulting argon concentration is $c \approx 15$ mM.

${ }^{b}$ The local concentration of argon in the filled pore reaches the much higher value of $415 \mathrm{mM}$, having used $14 \AA$ for the pore diameter and $26 \AA$ for the height.

\section{REFERENCES}

(1) Beckstein, O.; Sansom, M. S. Liquid-vapor oscillations of water in hydrophobic nanopores. Proc. Natl. Acad. Sci. U. S. A. 2003, 100, 7063-7068.

(2) Smeets, R. M.; Keyser, U.; Wu, M.; Dekker, N.; Dekker, C. Nanobubbles in solid-state nanopores. Phys. Rev. Lett. 2006, 97, No. 088101.

(3) Roth, R.; Gillespie, D.; Nonner, W.; Eisenberg, R. E. Bubbles, gating, and anesthetics in ion channels. Biophys. J. 2008, 94, 42824298.

(4) Anishkin, A.; Akitake, B.; Kamaraju, K.; Chiang, C.; Sukharev, S. Hydration properties of mechanosensitive channel pores define the energetics of gating. J. Phys.: Condens. Matter 2010, 22, 454120.

(5) Aryal, P.; Sansom, M. S.; Tucker, S. J. Hydrophobic gating in ion channels. J. Mol. Biol. 2015, 427, 121-130.

(6) Rao, S.; Lynch, C. I.; Klesse, G.; Oakley, G. E.; Stansfeld, P. J.; Tucker, S. J.; Sansom, M. S. Water and hydrophobic gates in ion channels and nanopores. Faraday Discuss. 2018, 209, 231-247.

(7) Hummer, G.; Rasaiah, J. C.; Noworyta, J. P. Water conduction through the hydrophobic channel of a carbon nanotube. Nature 2001, 414, 188-190.

(8) Falk, K.; Sedlmeier, F.; Joly, L.; Netz, R. R.; Bocquet, L. Molecular origin of fast water transport in carbon nanotube membranes: superlubricity versus curvature dependent friction. Nano Lett. 2010, 10, 4067-4073.

(9) Eroshenko, V.; Regis, R.-C.; Soulard, M.; Patarin, J. Energetics: a new field of applications for hydrophobic zeolites. J. Am. Chem. Soc. 2001, 123, 8129-8130.

(10) Amabili, M.; Grosu, Y.; Giacomello, A.; Meloni, S.; Zaki, A.; Bonilla, F.; Faik, A.; Casciola, C. Pore Morphology Determines Spontaneous Liquid Extrusion from Nanopores. ACS Nano 2019, 13, $1728-1738$

(11) Siwy, Z. S.; Powell, M. R.; Petrov, A.; Kalman, E.; Trautmann, C.; Eisenberg, R. S. Calcium-induced voltage gating in single conical nanopores. Nano Lett. 2006, 6, 1729-1734.

(12) Vlassiouk, I.; Siwy, Z. S. Nanofluidic diode. Nano Lett. 2007, 7, $552-556$.

(13) Vlassiouk, I.; Smirnov, S.; Siwy, Z. Ionic selectivity of single nanochannels. Nano Lett. 2008, 8, 1978-1985.

(14) Shi, W.; Friedman, A. K.; Baker, L. A. Nanopore sensing. Anal. Chem. 2017, 89, 157-188.

(15) Howorka, S.; Siwy, Z. Nanopore analytics: sensing of single molecules. Chem. Soc. Rev. 2009, 38, 2360-2384.

(16) Stoddart, D.; Heron, A. J.; Mikhailova, E.; Maglia, G.; Bayley, $\mathrm{H}$. Single-nucleotide discrimination in immobilized DNA oligonucleotides with a biological nanopore. Proc. Natl. Acad. Sci. U. S. A. 2009, 106, 7702-7707.

(17) Giacomello, A.; Roth, R. Bubble formation in nanopores: a matter of hydrophobicity, geometry, and size. Advances in Physics: $X$ 2020, 5, 1817780 .

(18) Li, W.; Zuo, X.; Zhou, X.; Lu, H. Effect of aggregated gas molecules on dewetting transition of water between nanoscale hydrophobic plates. J. Chem. Phys. 2019, 150, 104702.

(19) Smeets, R. M.; Keyser, U. F.; Dekker, N. H.; Dekker, C. Noise in solid-state nanopores. Proc. Natl. Acad. Sci. U. S. A. 2008, 105, 417-421.

(20) Franks, N. P. General anaesthesia: from molecular targets to neuronal pathways of sleep and arousal. Nat. Rev. Neurosci. 2008, 9, $370-386$.
(21) Pavel, M. A.; Petersen, E. N.; Wang, H.; Lerner, R. A.; Hansen, S. B. Studies on the mechanism of general anesthesia. Proc. Natl. Acad. Sci. U. S. A. 2020, 117, 13757.

(22) Sauguet, L.; Fourati, Z.; Prange, T.; Delarue, M.; Colloc'h, N. Structural basis for xenon inhibition in a cationic pentameric ligandgated ion channel. PLoS One 2016, 11, No. e0149795.

(23) Berendsen, H.; Grigera, J.; Straatsma, T. The missing term in effective pair potentials. J. Phys. Chem. 1987, 91, 6269-6271.

(24) Zhu, C.; Gao, Y.; Li, H.; Meng, S.; Li, L.; Francisco, J. S.; Zeng, X. C. Characterizing hydrophobicity of amino acid side chains in a protein environment via measuring contact angle of a water nanodroplet on planar peptide network. Proc. Natl. Acad. Sci. U. S. A. 2016, 113, 12946-12951.

(25) Verlet, L.; Weis, J.-J. Perturbation theory for the thermodynamic properties of simple liquids. Mol. Phys. 1972, 24, 1013-1024.

(26) Taylor, R. S.; Dang, L. X.; Garrett, B. C. Molecular dynamics simulations of the liquid/vapor interface of SPC/E water. J. Phys. Chem. 1996, 100, 11720-11725.

(27) Martyna, G. J.; Klein, M. L.; Tuckerman, M. Nosé-Hoover chains: the canonical ensemble via continuous dynamics. J. Chem. Phys. 1992, 97, 2635-2643.

(28) Tortora, M.; Meloni, S.; Tan, B. H.; Giacomello, A.; Ohl, C.-D.; Casciola, C. M. The Interplay Among Gas, Liquid and Solid Interactions Determines the Stability of Surface Nanobubbles. Nanoscale, in press, DOI: 10.1039/d0nr05859a.

(29) Maragliano, L.; Vanden-Eijnden, E. A Temperature Accelerated Method for Sampling Free Energy and Determining Reaction Pathways in Rare Events Simulations. Chem. Phys. Lett. 2006, 426, $168-175$.

(30) Harker, M.; O'Leary, P. Regularized reconstruction of a surface from its measured gradient field. J. Math. Imaging Vision 2015, 51, $46-70$.

(31) Tinti, A.; Giacomello, A.; Grosu, Y.; Casciola, C. M. Intrusion and extrusion of water in hydrophobic nanopores. Proc. Natl. Acad. Sci. U. S. A. 2017, 114, E10266-E10273.

(32) E, W.; Ren, W.; Vanden-Eijnden, E. Simplified and improved string method for computing the minimum energy paths in barriercrossing events. J. Chem. Phys. 2007, 126, 164103.

(33) Tinti, A.; Giacomello, A.; Casciola, C. M. Vapor nucleation paths in lyophobic nanopores. Eur. Phys. J. E: Soft Matter Biol. Phys. 2018, 41, 52.

(34) Lefevre, B.; Saugey, A.; Barrat, J.-L.; Bocquet, L.; Charlaix, E.; Gobin, P.-F.; Vigier, G. Intrusion and extrusion of water in hydrophobic mesopores. J. Chem. Phys. 2004, 120, 4927-4938.

(35) Guillemot, L.; Biben, T.; Galarneau, A.; Vigier, G.; Charlaix, É. Activated drying in hydrophobic nanopores and the line tension of water. Proc. Natl. Acad. Sci. U. S. A. 2012, 109, 19557-19562.

(36) Berg, H. C.; Purcell, E. M. Physics of chemoreception. Biophys. J. 1977, 20, 193-219.

(37) Kopp Lugli, A.; Yost, C. S.; Kindler, C. H. Anaesthetic mechanisms: update on the challenge of unravelling the mystery of anaesthesia. Eur. J. Anaesthesiol. 2009, 26, 807.

(38) Dickinson, R.; Franks, N. P. Bench-to-bedside review: Molecular pharmacology and clinical use of inert gases in anesthesia and neuroprotection. Crit. Care 2010, 14, 229.

(39) Oakes, V.; Domene, C. Capturing the Molecular Mechanism of Anesthetic Action by Simulation Methods. Chem. Rev. 2019, 119, 5998-6014.

(40) Jain, M.; Olsen, H. E.; Paten, B.; Akeson, M. The Oxford Nanopore MinION: delivery of nanopore sequencing to the genomics community. Genome Biol. 2016, 17, 239.

(41) Dekker, C. Solid-state nanopores. Nat. Nanotechnol. 2007, 2, 209.

(42) Bayley, H.; Braha, O.; Kasianowicz, J.; Gouaux, E. Designed protein pores as components for biosensors. US Patent Appl. 10/ 946,802, 2005.

(43) Giacomello, A.; Schimmele, L.; Dietrich, S.; Tasinkevych, M. Recovering superhydrophobicity in nanoscale and macroscale surface textures. Soft Matter 2019, 15, 7462-7471. 
(44) Eroshenko, V. Heterogeneous structure for accumulating or dissipating energy, methods of using such a structure and associated devices. US Patent 6,052,992, 2000.

(45) Eroshenko, V.; Regis, R.-C.; Soulard, M.; Patarin, J. The heterogeneous systems 'water-hydrophobic zeolites': new molecular springs. C. R. Phys. 2002, 3, 111-119. 